\title{
Atypical presentation of spinal intramedullary astrocytoma in a patient presenting with isolated signs of intracranial hypertension
}

\author{
Anthony Stefanelli $\mathbb{\circledR}^{1} \cdot$ Subhadra Acharya ${ }^{2} \cdot$ Victor Sabourin $^{1} \cdot$ Ritam Ghosh $^{1} \cdot$ Christopher Farrell $^{1}$
}

Received: 11 February 2020 / Revised: 14 March 2020 / Accepted: 16 March 2020

(c) International Spinal Cord Society 2020

\begin{abstract}
Introduction Intramedullary spinal tumors are rare entities that typically present with signs of spinal cord dysfunction including myelopathy, weakness, hypoesthesia, or bladder dysfunction. However, they can present in more insidious ways without signs of spinal cord dysfunction, as we will discuss in this case. Our patient presented with isolated signs of intracranial hypertension including headache and progressive vision loss. Although idiopathic intracranial hypertension (IIH) is significantly more common than spinal cord tumors, a full evaluation to rule out other diagnoses should always occur, as this is an exclusionary diagnosis.

Case presentation We describe an interesting case of an obese, middle-aged female who presented with signs and symptoms of elevated intracranial pressure (ICP) including progressive headache, visual changes, and papilledema. This led to a presumptive diagnosis of idiopathic intracranial hypertension (IIH). However, a careful review of her cerebrospinal fluid (CSF) analysis revealed a significantly elevated protein level. This prompted a more thorough workup, including a spinal MRI, that revealed an intramedullary astrocytoma. On her presenting physical exam, she had no upper or lower motor signs, no weakness, and no pathological reflexes.

Discussion In the evaluation of a patient with isolated signs of increased ICP concerning for IIH, it is important to conduct a thorough workup as this remains a diagnosis of exclusion. Many intracranial and intraspinal pathologies can manifest with similar vague neurological symptoms and masquerade as a more benign disease as shown in our patient who, unfortunately, died from a spinal astrocytoma.
\end{abstract}

\section{Introduction}

We describe an interesting case of an obese, middle-aged female who presented with signs and symptoms of elevated intracranial pressure (ICP) including progressive headache, visual changes, and papilledema. This led to an initial presumptive diagnosis of idiopathic intracranial hypertension (IIH). However, a careful review of her cerebrospinal fluid (CSF) analysis revealed a significantly elevated protein level. This prompted a more thorough workup, including a spinal MRI that revealed an intradural intramedullary

Anthony Stefanelli

anthony.stefanelli@jefferson.edu

1 Department of Neurological Surgery, Thomas Jefferson University Hospital, 900 Walnut Street, Philadelphia, PA 19107, USA

2 Sidney Kimmel Medical College, Thomas Jefferson University, 1025 Walnut St \#100, Philadelphia, PA 19107, USA astrocytoma at T11-T12. On her presenting physical exam, she had no upper or lower motor signs, no weakness, and no pathological reflexes. Although IIH is significantly more common than spinal cord astrocytomas, a full evaluation to rule out other diagnoses should always occur.

\section{Case presentation}

A 45-year-old morbidly obese Bengali female with no significant past medical history presented to an outside hospital with $\sim 6$ weeks of progressive vision changes, headaches, nausea, and vomiting. She noticed an acute and significant decline in her vision $\sim 3-4$ days prior to her initial presentation that had not improved and led to the outside hospital emergency department visit.

Physical exam demonstrated bilateral papilledema, a mild right abducens nerve (CN VI) palsy, and anisocoria (right pupil $6 \mathrm{~mm}$ and reactive, left pupil $7 \mathrm{~mm}$ and sluggish). She had no other focal neurological deficits. She had 


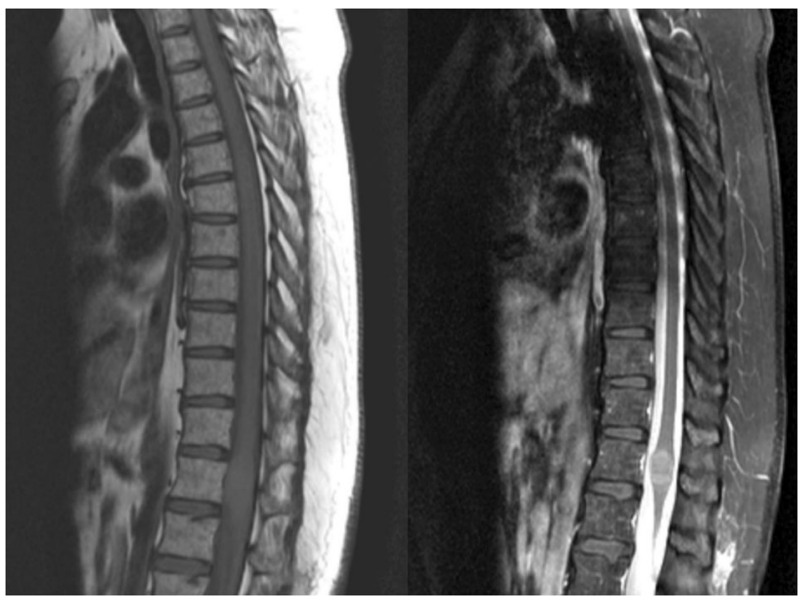

Fig. 1 Thoracic Spine MRI. T1 MRI without gadolinium (left) and T1 MRI with gadolinium (right) demonstrating an expansile heterogeneously enhancing thoracic intramedullary lesion with grossly enhancing leptomeninges.

a brain MRI, MRA, and MRV that revealed no intracranial mass lesion, infarct, or venous sinus thrombosis. She underwent a lumbar puncture at the outside hospital and was found to have an opening pressure of $45 \mathrm{~cm} \mathrm{H}_{2} \mathrm{O}$. CSF protein was found to be significantly elevated at $950 \mathrm{mg} / \mathrm{dL}$ with an otherwise unremarkable profile. She was started on acetazolamide therapy and transferred to our institution for evaluation for placement of a ventriculoperitoneal shunt, as the outside hospital did not have neurosurgical coverage.

At our institution, a more thorough workup was conducted due to the significantly elevated CSF protein levels, which is not typical of IIH. The patient underwent a spinal MRI with and without gadolinium, which revealed a $17.5 \mathrm{~mm}$ heterogeneously enhancing intramedullary mass at T11-12 with significant subarachnoid seeding concerning for leptomeningeal carcinomatosis (Fig. 1).

To relive the symptoms of hydrocephalus and obtain a diagnosis, the patient underwent placement of a ventriculoperitoneal shunt as well as an L4 laminectomy and open biopsy of the enhancing dura. Neuropathology identified the tissue as containing atypical glial fibrillary acidic protein positive cells suspicious for neoplasm; however, the final pathology was undiagnostic. The patient had been subsequently discharged to a rehabilitation facility prior to obtaining the final pathology results.

Approximately 2 weeks later, she presented back to the hospital with lower extremity weakness and signs of shunt malfunction necessitating a proximal and distal shunt revision along with a T11-L1 laminectomy for open biopsy of the thickened arachnoid and lesion. Final pathology was consistent with a highly proliferative astrocytic glial neoplasm with a markedly elevated Ki-67 index of $26 \%$, which represents a measure of cellular proliferation in neoplasia. After being discharged, she returned for a second shunt

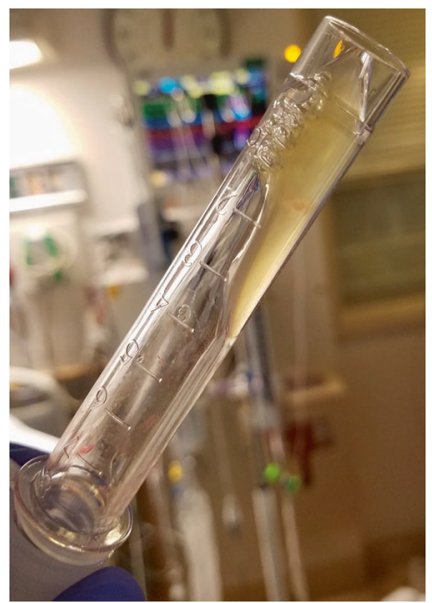

Fig. 2 CSF Specimen. Specimen tube demonstrating honey thick CSF with elevated protein of $2700 \mathrm{mg} / \mathrm{dL}$.

revision and placement of Ommaya reservoir for infusion of intrathecal chemotherapy. Despite these interventions, her clinical exam deteriorated over the following weeks. She ultimately presented back to the emergency department following a generalized tonic-clonic seizure in ICP crisis and therefore underwent emergent placement of an external ventricular drain. Her CSF protein continued to increase to $2700 \mathrm{mg} / \mathrm{dL}$ with a honey thick consistency (Fig. 2). This made CSF diversion difficult and patient continued to have ICP crises. Despite maximal medical management, the patient ultimately herniated and went into cardiopulmonary arrest. Family chose to proceed with a Do Not Resuscitate order at this time and the patient died shortly afterwards.

\section{Discussion}

In the evaluation of a patient with isolated signs of increased ICP concerning for IIH, it is important to conduct a thorough workup as this remains a diagnosis of exclusion. Many intracranial and intraspinal pathologies can manifest with similar vague neurological symptoms and masquerade as a more benign disease. The differential diagnosis is vast for these patients and includes migraine headache, venous sinus thrombosis, cerebral infarct/hemorrhage, metabolic derangements, chemical toxicity, autoimmune conditions, as well as tumors of the brain or spine.

In this case, our patient presented with symptoms that were most suspicious of IIH. She fit the typical demographic (a middle-aged obese female) with consistent presenting symptoms. Her cranial imaging demonstrated no pathological process, such as a tumor or sinus thrombosis, that otherwise could have explained her presentation. Her exam also demonstrated no signs of myelopathy, lower extremity weakness, or pathological reflexes. However, her 
significantly elevated CSF protein level was concerning for something more insidious and ultimately prompted us to obtain spinal imaging which led to the diagnosis of an intramedullary astrocytoma.

In general, spinal astrocytomas are rare entities, with only $3 \%$ occurring in the spinal cord compared with the brain [1]. These tumors tend to involve multiple spinal levels with up to $20 \%$ associated with a syrinx [2]. They have an association with Neurofibromatosis type 1 , occur predominantly in males, and tend to manifest in patients under the age of 60 [3]. Although uncommon, spinal astrocytomas and other intramedullary tumors can present with isolated signs of elevated ICP without other symptoms due to possible interference with CSF reabsorption [4].

Upon review of the literature, several similar, but unique, cases have been described. Porter et al cited the case of a young man who presented with increased ICP, papilledema, and obscured vision, which initially led to an incorrect diagnosis of IIH [5]. This patient's CSF protein level was only slightly elevated, and the patient was treated as though he had IIH. He developed myelopathy 7 months after initial diagnosis, prompting an MRI that identified an intramedullary mass from C7-T4, later found to be an astrocytoma. This case resembles our patient in that she had no initial signs of myelopathy or weakness.

Costello et al. noted the case of a woman who presented with papilledema and signs of increased ICP as the initial manifestations of a spinal schwannoma [6]. As well, Miranda Bacallado et al. describe a young man who presented with intracranial hypertension and CN VI palsy as the initial symptoms of a conus medullaris ependymoma [7].

In summary, intramedullary spinal tumors are a rare cause of isolated increased ICP, especially in the absence of other symptoms such as myelopathy or upper motor signs. However, our case is not isolated, reinforcing the importance of a thorough workup to prevent delay in diagnosis of another more sinister pathology.

\section{Compliance with ethical standards}

Conflict of interest The authors declare that they have no conflict of interest.

Publisher's note Springer Nature remains neutral with regard to jurisdictional claims in published maps and institutional affiliations.

\section{References}

1. Sloof JL, Kernohan JW, MacCarthy CS. Primary intramedullary tumors of the spinal cord and filum terminale. Philadelphia, PA: Saunders; 1964.

2. Samii M, Klekamp J. Surgical results of 100 intramedullary tumors in relation to accompanying syringomyelia. Neurosurgery. 1994:35:865-73.

3. Minehan KJ, Shaw EG, Scheithauer BW, Davis DL, Onofrio BM. Spinal cord astrocytoma: pathological and treatment considerations. J Neurosurg. 1995;83:590-5.

4. Rekate HL. Why would a spinal tumor cause increased intracranial pressure? J Neuroophthalmol. 2002;22:197-8.

5. Porter A, Lyons MK, Wingerchuk DM, Bosch EP. Spinal cord astrocytoma presenting as "idiopathic" intracranial hypertension. Clin Neurol Neurosurg. 2006;108:787-9.

6. Costello F, Kardon RH, Wall M, Kirby P, Ryken T, Lee AG. Papilledema as the presenting manifestation of spinal schwannoma. J Neuroophthalmol. 2002;22:199-203.

7. Miranda Bacallado Y, González Hernández A, Curutchet Mesner L, Rúa-Figueroa Fernández, de Larrinoa I. Bilateral papilloedema as the initial manifestation of spinal ependymoma. Neurologia. 2018;33:194-6. 